\title{
Nesprins: from the nuclear envelope and beyond
}

\section{Dipen Rajgor and Catherine M. Shanahan*}

Nuclear envelope spectrin-repeat proteins (Nesprins), are a novel family of nuclear and cytoskeletal proteins with rapidly expanding roles as intracellular scaffolds and linkers. Originally described as proteins that localise to the nuclear envelope (NE) and establish nuclear-cytoskeletal connections, nesprins have now been found to comprise a diverse spectrum of tissue specific isoforms that localise to multiple sub-cellular compartments. Here, we describe how nesprins are necessary in maintaining cellular architecture by acting as essential scaffolds and linkers at both the NE and other sub-cellular domains. More importantly, we speculate how nesprin mutations may disrupt tissue specific nesprin scaffolds and explain the tissue specific nature of many nesprin-associated diseases, including laminopathies.

The eukaryotic cytoplasm contains three major types of cytoskeletal filaments: Filamentousactin (F-actin), microtubules (MTs) and intermediate filaments (IFs). These components are organised in a manner that provides the cell with an internal framework fundamental for many processes, such as controlling cellular shape, polarity, adhesion and migration, cytokinesis, inter- and intracellular communication and trafficking of organelles, vesicles, proteins and RNA (Refs 1, 2).

For cells to utilise the cytoskeleton in these processes, scaffold or linker proteins are required to attach cytosolic components to these dynamic filaments. The spectrin-repeat (SR) family of proteins are a prime example of such linkers. The majority of family members are typically characterised by a pair of N-terminal calponin homology domains (CHDs) that bind F-actin, followed by a rod-like structure composed of multiple SRs. A single SR is composed of three $\alpha$-helical bundles with a lefthanded twist, and its primary function is to provide docking sites for proteins and other higher order complexes (Refs 3, 4). Although most SR proteins contain CHDs, some possess motifs which can interact with other cytoskeletal components, allowing linkage of SR-associated complexes to filamentous structures other than F-actin. In addition, these motifs allow crosslinking between different filaments and dynamic re-modelling of the cells internal framework in response to altered mechanical needs (Refs 5, 6, $7)$. The SR family is composed of a growing list of proteins that include $\alpha$-actinins, $\alpha / \beta$ spectrins, dystrophins and spectraplakins. Family members are distinguished by unique structural domains, which give the individual family members their specialised functions. For example, the C-terminal Pleckstrin Homology domain of $\beta$-spectrins facilitate membrane attachment to the cell surface and organelles, the

James Black Centre, Cardiovascular Division, King's College London, 125 Coldharbour lane, London SE5 9NU, UK

${ }^{*}$ Corresponding author: C.M. Shanahan, James Black Centre, Cardiovascular Division, King's College London, 125 Coldharbour lane, London SE5 9NU, UK. E-mail: cathy.shanahan@kcl.ac.uk

Accession information: doi:10.1017/erm.2013.6; Vol. 15; e5; July 2013 (c) Cambridge University Press 2013. Re-use permitted under a Creative Commons Licence - by-nc-sa. 
WW and Zn-fingers of dystrophins associate with the dystroglycan complex spanning the plasma membrane to provide extracellular matrix attachment and the GAR domains of spectraplakins associate with MTs and act as cytoskeletal cross-linkers (Refs 8, 9, 10, 11, 12, 13).

Nuclear envelope spectrin-repeat proteins (Nesprins), are the latest members of the SR super family to be identified. They contain a unique evolutionary conserved C-terminal Klarsicht, ANC-1 and Syne Homology (KASH) transmembrane domain that specifically recruits the nesprins to the nuclear envelope (NE). To date, four separate nesprin genes have been identified in vertebrates, which encode proteins with distinct cellular functions. Nesprin-1 and nesprin-2 are the largest SR proteins with molecular weights of $\sim 1000$ and $\sim 800 \mathrm{kDa}$, respectively, and connect the NE to F-actin through their pair of N-terminal CHDs. Nesprin-3 and nesprin-4, with molecular weights of $\sim 110$ and $\sim 42 \mathrm{kDa}$, respectively, lack N-terminal CHDs (Refs 14, 15, 16, 17). Instead, nesprin-3 contains a plectin-binding domain which links the NE to the cytoplasmic IF networks. Nesprin-4 associates with kif5b, a subunit of the MT motor protein kinesin-1, downstream of its single SR (Refs 16, 17) (Fig. 1). The ability of the nesprins to localise to the NE and interact with cytoskeletal proteins, initially led to the assumption that their primary purpose was to act as cytoskeletal-NE linkers. Excitingly, recent developments in nesprin biology have identified an expansive collection of tissue specific nesprin-1 and nesprin-2 isoforms generated through alternative transcription (Ref. 18). Many of these isoforms exclude the C-terminal KASH domain and localise to various sub-cellular compartments, supporting additional functions for nesprins other than NE linkage.

This review focuses on summarising recent findings in which scaffolding roles for nesprins have been identified at the NE, and beyond. Furthermore, we speculate that many of the tissue specific diseases resulting from nesprin mutations, including cardiac and skeletal muscle dysfunctions in Emery Dreifuss Muscular Dystrophy (EDMD), cerebellar defects in autosomal recessive cerebellar ataxia 1 (ARCA1), tendon contractures in autosomal recessive arthrogryposis multiplex congenita (AMC), cardiac defects in dilated cardiomyopathy (DCM) and major depression in bipolar disease, maybe a consequence of defects in the production or scaffolding functions of a subset of tissue specific nesprin isoforms, and thus, in some instances, be completely independent of NE-cytoskeletal linkage (Refs 19, $20,21,22,23)$.

\section{Nesprins link the NE to the cytoskeleton and nuclear lamina}

The nucleus is surrounded by a system of two concentric membranes, called the inner nuclear membrane (INM) and the outer nuclear membrane (ONM). Together, these two membranes make up the NE and separate the
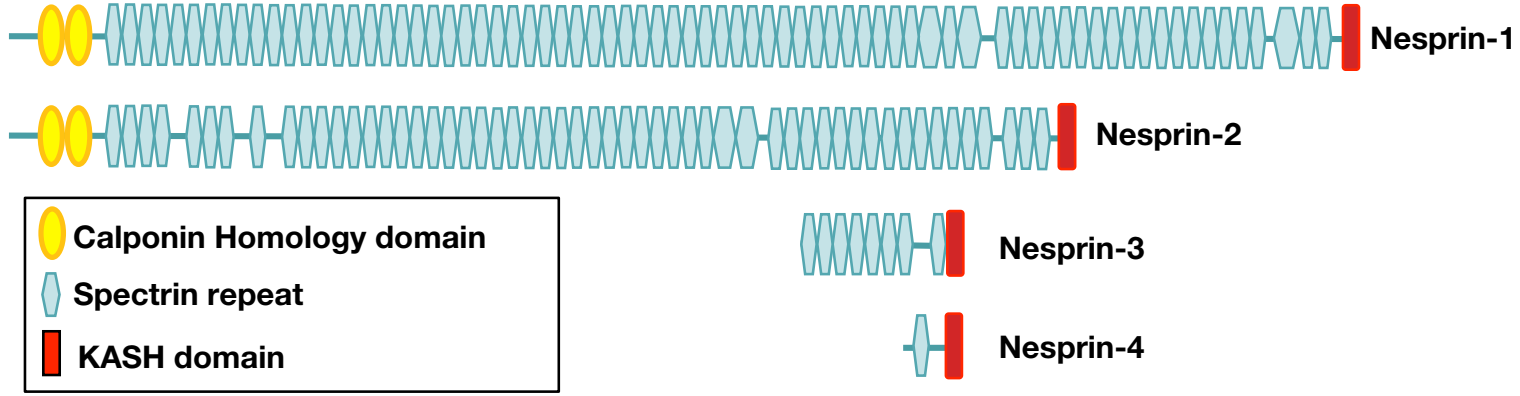

Nesprin-2

Figure 1. The nesprin family. The nesprin family is composed of four members, all of which contain a Cterminal nuclear envelope (NE) targeting Klarsicht, ANC-1 and Syne Homology (KASH) domain, and one or more spectrin-repeats (SRs). Nesprin-1 contains $74 \mathrm{SRs}$ and binds to F-actin through its $\mathrm{N}$-terminal calponin homology domains (CHDs). The shorter nesprin-2 protein is composed of 56 SRs, and like nesprin-1 is able to bind F-actin through its N-terminal CHDs. Nesprin-3 contains 8 SRs and interacts with the versatile cytoskeletal cross-linker plectin in a manner that allows nesprin-3 to associate with intermediate filaments (IFs). Nesprin-4 possesses only a single SR and interacts with kif5b, a subunit of the microtubule (MT) motor kinesin-1.

Accession information: doi:10.1017/erm.2013.6; Vol. 15; e5; July 2013 (c) Cambridge University Press 2013. Re-use permitted under a Creative Commons Licence - by-nc-sa. 
cells nuclear contents from the cytosol. Although the ONM and INM are a single continuous joint membrane, perforated by nuclear pore complexes and separated by a lumen, macromolecular compositions of the two sides vary. The ONM has a similar composition to that of the adjoined endoplasmic reticulum, with the addition of full-length nesprins-1, -2, -3 and -4 that link the NE to components of the cytoskeleton (Refs 15, 16, 17). The INM hosts a unique collection of transmembrane proteins, which link the INM to the underlying nuclear lamina and chromatin (Refs 24, 25, 26, 27, 28).

At the NE, the C-terminal sequence of nesprin $\mathrm{KASH}$ domains bind to the C-terminal end of the trimeric INM SUN (Sad1p-UNC-84) domain proteins, SUN1 and SUN2, within the NE lumen (Refs 29, 30). The N-terminal tail of the trimeric SUN proteins interacts with the nuclear lamina underlying the nuclear interior (Refs 29, 31, 32). The nuclear lamina, composed of type-V IF lamin proteins, forms a mechanical fibrous cage around the interior surface of the NE which provides structural support to the nucleus and scaffolds nuclear signalling proteins, transcription factors and chromatin (Refs 24, 27, 28). This double membrane spanning complex, mediated by SUN-KASH bridges, links the cytoskeleton to the nucleoskeleton and is referred to as the 'linker of nucleoskeleton to cytoskeleton' (LINC) complex (Refs 30, 31, 32, 33, 34, 35) (Fig. 2). Small nesprin KASH variants generated through alternative transcription, such as nesprin- $1 \alpha$ and nesprin- $2 \beta$, are potentially capable of NE entry and are embedded within the INM. At the INM, their SRs project towards the nuclear lamina where they interact strongly with lamin $\mathrm{A} / \mathrm{C}$, emerin and signal transducers including mAKAP (Refs 19, 36, 37, 38). Rather than binding to the C-terminal SUN domain of the SUN proteins, these short KASH variants are likely to interact with sequences closer to the N-terminus, as shown with nesprin-2, which appears to have an overlapping binding site with emerin in SUN1 (Refs 31, 39).

Through hetero- and homo-oligomerisation events that occur between the SUN domains and nesprin proteins, LINC complexes form higher order structures that are more complex than originally anticipated. Recent crystallisation studies illustrate that the SUN domain of SUN2 forms a trimeric structure and interacts with 3 independent $\mathrm{KASH}$ proteins in a 3:3 stoichiometry ratio (Ref. 29). Although these oligomeric structures are yet to be proved in vivo, hypothetically any one of the many ONM nesprin variants (see below) could associate in any combination with a trimer of SUN domain proteins, allowing a large and mixed plethora of LINCs to form.

Nesprin-1 $\alpha$, which is enriched in cardiac, skeletal and smooth muscle, forms anti-parallel homodimers within the INM (Ref. 36). The significance of this dimerisation event remains unclear, but may provide additional mechanical strength to nuclei that are constantly exposed to contractile strain, by forming complex connections with the underlying nuclear lamina and other interacting partners.

In addition, nesprin-1 and nesprin- 2 giants (i.e. full-length nesprin-1 and nesprin-2 isoforms) dimerise with nesprin-3 at the ONM to form a meshwork composed of peri-nuclear cytoskeletal filaments around the nuclear periphery, which becomes disorganised when nesprin LINCS are disrupted (Refs 40, 41, 42). This meshwork may supply the necessary framework and act as a cushion for LINC complex functions that put considerable strain on the nucleus through force transmission. For example, the meshwork may function as a buffer from physical forces generated through nuclear migration or transduction of mechanical stimuli generated within the cell or from the extracellular environment. Besides nuclear migration and mechanotransduction, nesprin LINC complexes are required for cellular differentiation, synchronising the localisation of cytosolic organelles, aiding in establishing cell polarity and participating in cell migration, suggesting that they are central for precise coordination and organisation of the entire cell (Refs 17, 35, 42, 43, 44, 45, 46, 47, 48, 49, 50, 51, $52,53)$. Indeed, emerging evidence now strongly supports the cell as a structurally coupled system, with perturbations in mechanical stimuli influencing nuclear organisation, epigenetics and mechanotransduction through nesprin LINC complexes (Refs 54, 55, 56, 57).

\section{Heterogeneity of nesprins in the LINC complex}

Hypothetically, up to 16 nesprin- 1 and 12 nesprin$2 \mathrm{KASH}$ isoforms can be generated by alternatively combining identified 5'UTRs with the $3^{\prime}$ UTRs of the nesprin-1 and nesprin-2 giant full-length

Accession information: doi:10.1017/erm.2013.6; Vol. 15; e5; July 2013 (C) Cambridge University Press 2013. Re-use permitted under a Creative Commons Licence - by-nc-sa. 


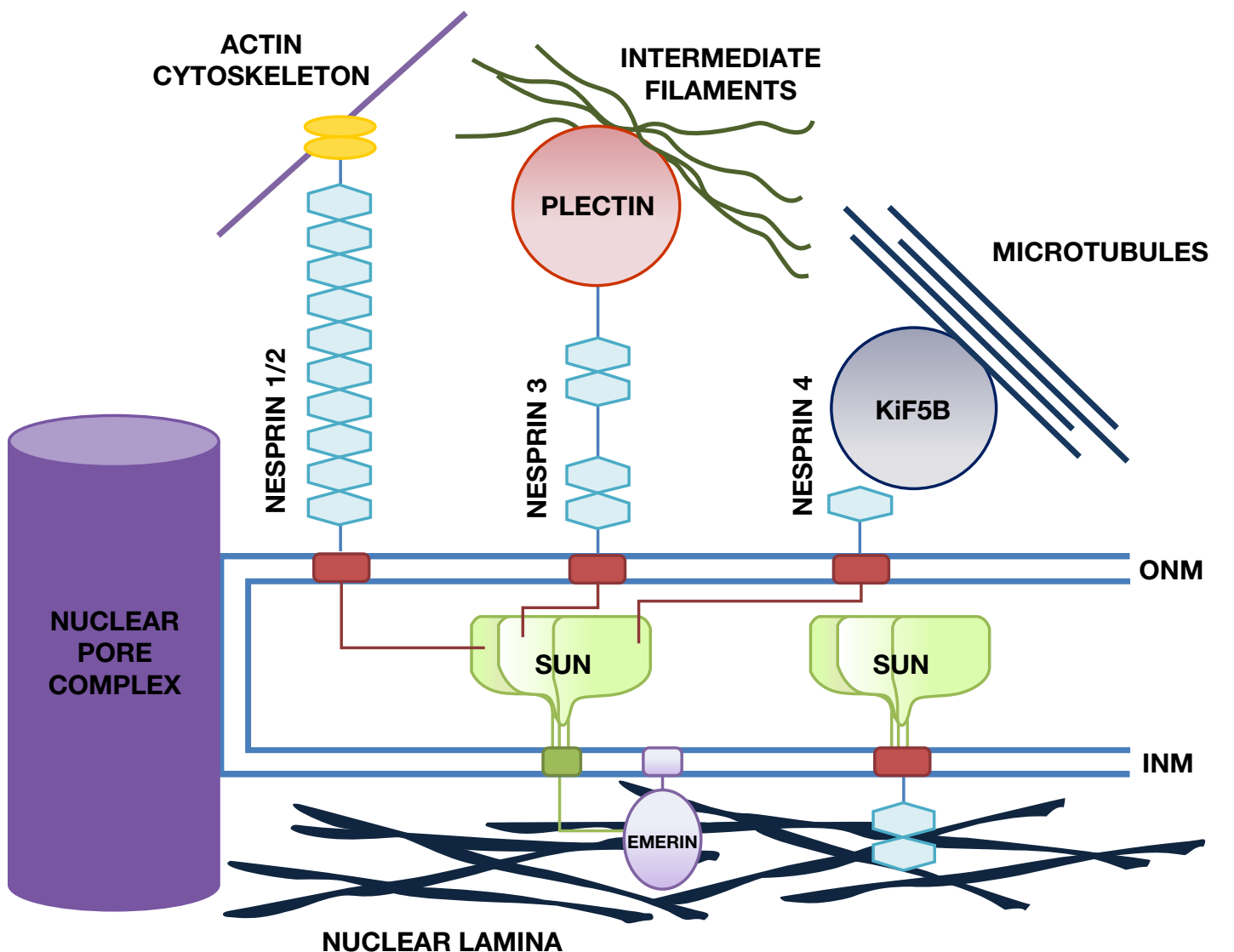

Figure 2. Nesprins link the nucleoskeleton to components of the cytoskeleton. The linker of nucleoskeleton to cytoskeleton (LINC) complexes couples the nuclear lamina to cytoskeletal networks via the nesprins. The LINC complex is comprised of SUN (Sad1p-UNC-84) domain containing proteins, which exist as trimers, in the inner nuclear membrane (INM) and associate with the nuclear lamina via interactions with the lamin proteins, emerin and small INM nesprin isoforms. The Klarsicht, ANC-1 and Syne Homology (KASH) domains of full-length nesprins-1, nesprin-2, nesprin-3 and nesprin-4 in the outer nuclear membrane (ONM) bind to the SUN domains of the SUN proteins in a 3:3 KASH:SUN ratio within the luminal space, and serve to tether the nuclear envelope (NE) to cytoplasmic F-actin, intermediate filaments (IFs) or microtubules (MTs) respectively. Small KASH isoforms present within the INM do not interact with the SUN domain of SUN protein. Instead, they appear to interact with the emerin binding region in SUN proteins via their spectrin repeats (SRs). NE-cytoskeletal interactions via the LINC complex are accountable for nuclear anchorage, nuclear positioning, nuclear migration, cell polarity, cell differentiation, mechanotransduction, nuclear cytoskeletal organisation and organelle positioning.

variants, which terminate after inclusion of the KASH domain (Fig. 3). Interestingly, the recently identified tissue specific expression of nesprin 5'UTRs implies that different cells and tissues are likely to express different KASH isoforms; although expression of many of the large variants need to be validated (Ref. 18). Early Northern blotting experiments using probes to target the C-terminal region of nesprin-1 and nesprin-2 detected multiple sized transcripts in a tissue specific manner, supporting the existence of multiple KASH isoform transcripts (Refs 14,
58). This is consistent with proteomic analysis demonstrating variations in the NE proteome in different tissues (Ref. 26). Putative tissue specific nesprin KASH isoforms vary in size from $\sim 53 \mathrm{kDa}$ of p53KASH ${ }^{\mathrm{Nesp} 1}$ to $\sim 1000 \mathrm{kDa}$ of the nesprin-1 giant for nesprin-1, and $\sim 48 \mathrm{kDa}$ of nesprin- $2 \alpha_{2}$ to $\sim 800 \mathrm{kDa}$ of the nesprin-2 giant for nesprin-2. The large $\mathrm{KASH}$ isoforms are expected to localise at the ONM because of size restriction of protein transport across the NE, however the smaller variants are probably capable of embedding within either membrane. 
30

40

50

60

70

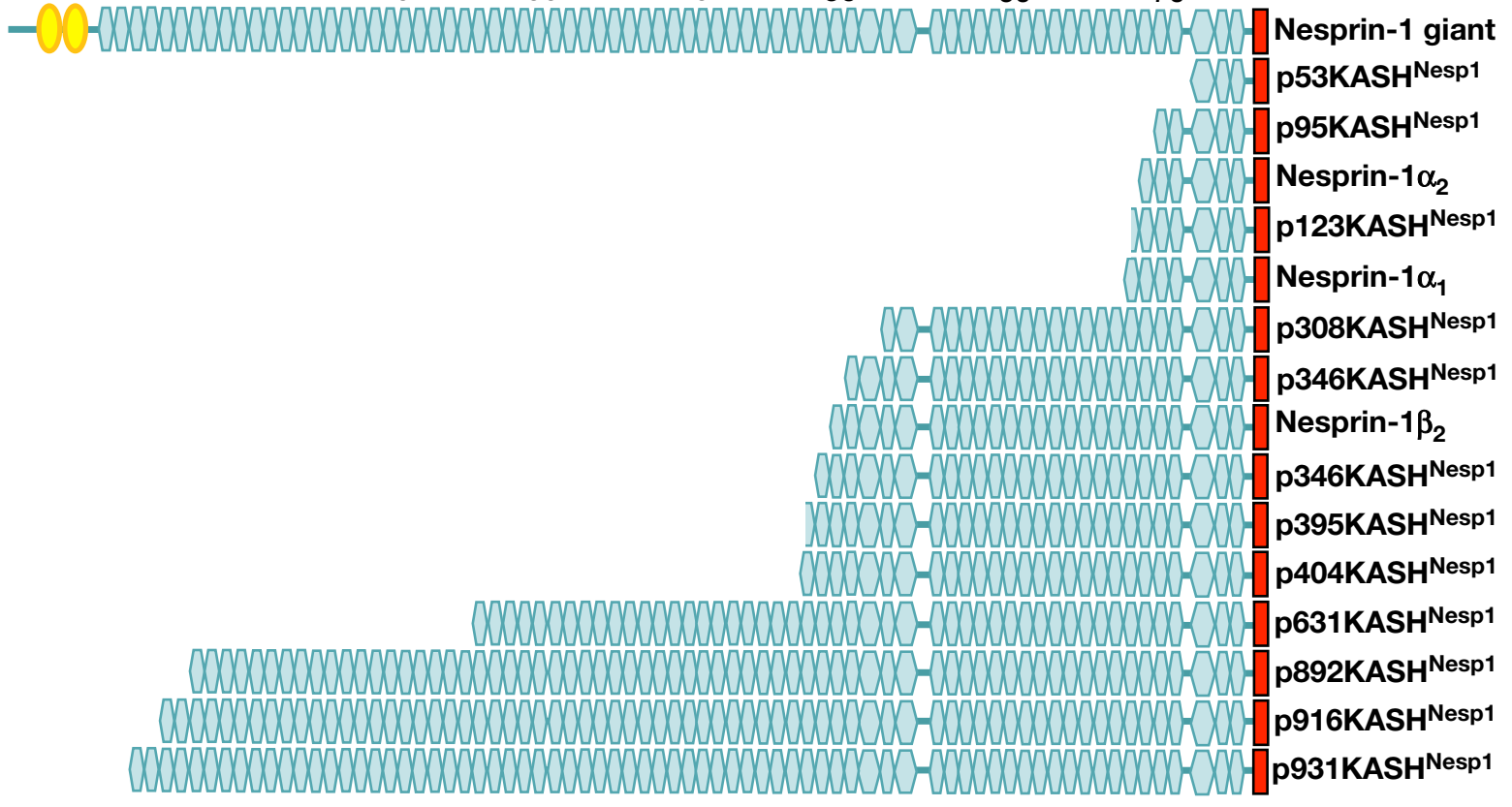

Nesprin-1 giant p53KASH ${ }^{\text {Nesp1 }}$ p95KASH ${ }^{\text {Nesp1 }}$ Nesprin-1 $\alpha_{2}$ Nesprin-1 $\alpha_{1}$

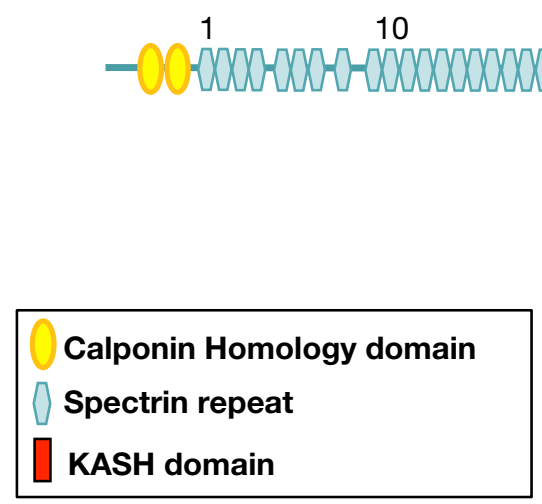

20

30

40

50

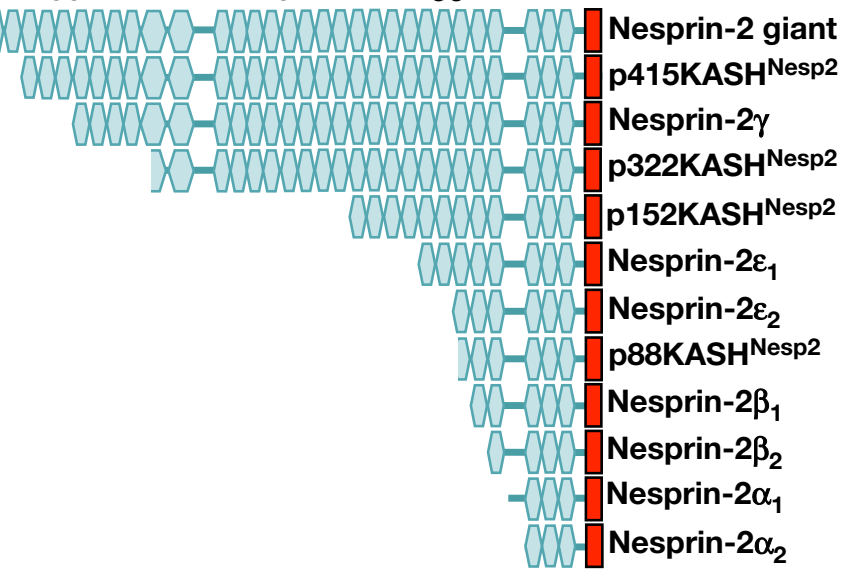

Nesprin-2 giant p415KASH ${ }^{\text {Nesp2 }}$ Nesprin-2 $\gamma$ p322KASH ${ }^{\mathrm{Nesp} 2}$ p152KASH ${ }^{\text {Nesp2 }}$ Nesprin-2 $\varepsilon_{1}$ Nesprin-2 $\varepsilon_{2}$

Figure 3. Potential nesprin-1 and nesprin-2 Klarsicht, ANC-1 and Syne Homology (KASH) variants. By combining all the identified $5^{\prime}$ UTRs with the nesprin-1 giant and nesprin-2 giant $3^{\prime}$ UTRs, up to 16 different nesprin-1 and 12 different nesprin-2 KASH domain-containing variants can be created. Although many of the large variants are hypothetical and yet to be validated at the mRNA and protein level, the tissue specific expression of the $5^{\prime}$ UTRs suggests that they are likely to be highly tissue specific. Furthermore, the exposure of unique $\mathrm{N}$-terminal binding motifs generated through alternative initiation suggests that each variant may scaffold unique protein complexes to the nuclear envelope (NE).

KASH isoforms that lack the CHDs may expose additional isoform specific N-terminal SR binding motifs, which could tether transcription factors or other nuclear signalling proteins for INM isoforms, or cytoskeletal components such as motor proteins for ONM isoforms. In addition, nesprins may also participate in tethering organelles to the nuclear membrane and in organelle organisation (Ref. 53). Theoretically, any three KASH isoforms could form bridges in a variety of different combinations with a single SUN domain trimer. Together, these nesprins could function in tethering tissue specific components to the NE, determined by a cells unique nesprin-KASH isoform profile and their SUN-KASH 
combinations. Their unique anchoring functions may influence or adapt nuclear mechanical stability, stiffness, positioning and migration, as well as cell polarity and cellular signalling pathways for tissue specific functions.

Moreover, nesprin isoform profiles are dynamically regulated during cellular differentiation and could be fundamental in developmental processes. For example, a switch from larger $\mathrm{KASH}$ isoforms to smaller $\mathrm{KASH}$ isoforms is observed during human myotube differentiation (Ref. 48). This may serve to alter LINC complex functions, enabling cells to change from a dynamic motile phenotype to a rigid stable contractile phenotype. During embryonic stem (ES) cell differentiation, the NE lumen becomes narrower in differentiated cells and nesprin-1 KASH isoforms become significantly upregulated. Although ES cells did not fail to differentiate when depleted of nesprin-1 using siRNA, their NE lumen failed to narrow and remained similar in size to their undifferentiated counterparts. This data suggests that dynamic regulation of nesprin-1 KASH isoforms acts to regulate NE structure during ES cell differentiation and could be important for maintaining their pluripotent state (Ref. 59).

\section{Nesprins in nuclear anchorage, positioning and migration}

Early insights into cytoskeletal-nuclear connections came from studying nesprins in lower organisms such as Caenorhabditis elegans and Drosophila melanogaster, which both only posses one nesprin-1/-2 homologue; ANC-1 and MSP-300 respectively. Mutations in these nesprin homologues or overexpression of dominant negative constructs affect nuclear anchorage in the hypodermal syncytia of $C$. elegans and nurse cells of D. melanogaster, demonstrating the importance of actin-LINCs in nuclear scaffolding (Refs 53, 60, 61).

In mammals, mouse models have been utilised to demonstrate that nuclear anchorage is mediated by nesprins in vivo. Transgenic mice overexpressing the dominant negative nesprin-1 KASH domain under the control of a muscle-specific promoter, display a loss of synaptic nuclear anchorage beneath the neuromuscular junction in skeletal muscle (Ref. 43). Nesprin-1 $1^{-/-K A S H}$ domain knockout $(\mathrm{KO})$ mice also demonstrate synaptic nuclei displacement, as well as additional aggregation of extra-synaptic nuclei. Furthermore, these $\mathrm{KO}$ mice display extensive branching of phrenic nerves, suggesting that nesprin-1 mediated synaptic nuclear anchorage may be essential for maintaining neuronal innervation sites. On the contrary, nesprin-2 ${ }^{-/-K A S H}$ domain $\mathrm{KO}$ mice do not appear to have any nuclear anchorage defects or notable phenotypes. However, when all actin-LINCs are abolished in nesprin-1 ${ }^{-/-\mathrm{KASH}}$ domain and nesprin-2-/-KASH domain double KOs, mice die at birth because of respiratory failure (Ref. 44). These data suggest that there may be some redundancy between nesprin-1 and nesprin-2 KASH isoforms, however the overlapping similarities are yet to be investigated.

Although these studies primarily assumed that disruption of actin-LINCs mediated through nesprin-1 and nesprin-2 giants were responsible for the accompanying phenotypes, they do not take into consideration the scaffolding function of the other KASH isoforms described in this review that would also be disrupted (Fig. 3). Consequently, these animal models may additionally disrupt $\mathrm{KASH}$ isoforms that do not form links exclusively with actin. For example, the interaction between nesprin-1 and nesprin-2 with dynein and kinesin-1 couples the centrosome to the neuronal nucleus in the developing mouse brain. In nesprin- $1^{-/-\mathrm{KASH}}$ domain and nesprin-2-1-KASH domain double $\mathrm{KO}$ mice, the centrosome is uncoupled from the neuronal nuclei, resulting in severe nucleokinesis and interkinetic nuclear migration defects (Ref. 62). Currently, the binding motifs for these motor proteins have not been mapped within the nesprin-1 and nesprin-2 proteins. They could interact with the giant full-length nesprins, or alternatively associate through one of the N-terminal SRs exposed in any of the truncated KASH domain variants (Fig. 3). Whereas nesprin-4 is only expressed in a selective population of epithelial cells to drive nuclear migration, alternate nesprin-1 and/or nesprin-2 KASH isoforms could perform similar functions in other cell types.

Interestingly, an SR in the central rod of nesprin1 also binds to kif3b, a kinesin-2 subunit, and appears to play a role in cytokinesis and membrane transport (Ref. 63). Whether this interaction occurs in specific KASH containing nesprin-1 variants or KASH-less variants still requires further investigation, but could link

Accession information: doi:10.1017/erm.2013.6; Vol. 15; e5; July 2013 (C) Cambridge University Press 2013. Re-use permitted under a Creative Commons Licence - by-nc-sa. 
nesprin-1 KASH variants in regulating cytokinesis.

\section{Organelle anchorage and positioning}

As well as being key in nuclear anchorage and positioning, nesprin LINCs have been implicated in the anchorage and positioning of other cytoplasmic organelles. For example, ANC-1 mediated actin-LINCs in C. elegans have been identified as mitochondrial scaffolds, where ANC-1 mutants display defective mitochondrial positioning (Ref. 53). In addition, overexpression of a dominant negative nesprin-1 Golgi-binding domain causes the Golgi to collapse into condensed structures near the centrosome in epithelial cells (Ref. 64). However, whether the correct anchorage of these organelles is mediated by LINC complex nesprins, LINC complex-independent nesprin isoforms, or a combination of both still remains unknown.

\section{KASH-less nesprin variants localise to multiple subcellular compartments}

The existence of nesprin variants in sub-cellular compartments other than the NE was first observed when antibodies were generated to different domains of nesprin-1 and nesprin-2. The currently available nesprin- 1 and nesprin-2 antibodies localise nesprins to the nucleolus, stress fibres, focal adhesions, Golgi, Centrosome, nuclear matrix, heterochromatin, sarcomere, plasma membrane, promyelocytic (PML) bodies, mitochondria and sarcoplasmic reticulum (Refs 14, 15, 36, 58, 63, 64, 65, 66, 67, 68, 69). Interestingly, some of these localisations are only observed in specific cell lines, indicating that the variants localising to these cellular sites are potentially tissue specific. Furthermore, these variants are likely to lack the KASH domain and be generated through the alternative combination of the nesprin-1 and nesprin-2 5'UTRs and 3'UTRs. The assortment of nesprin-1 and nesprin-2 transcripts produced through alternative transcription encode a diverse range of KASH-less variants, which can be further complicated when alternative splicing of the several cassette exons spread across the nesprin genes are taken into account (Ref. 18). Interestingly, many of these variants are transcribed in a tissue specific manner and appear to have different sub-cellular localisations within different cell lines when ectopically expressed. These data support the cell-specific staining observed with the nesprin antibodies and suggests that individual isoforms may mediate tissue-specific scaffolding and signalling events.

\section{CHD isoforms}

As with the KASH domain nesprin variants, multiple $\mathrm{CHD}$ variants can be generated by combining the full-length nesprin- 1 and nesprin- 2 giant 5'UTRs with the alternative $3^{\prime}$ UTRs spread across these genes. Alternative transcription would allow the generation of 14 putative nesprin- 1 and 5 putative nesprin-2 CHD isoforms, respectively, although expression of many of the large variants need to be validated (Fig. 4). Given the tendency of the CHDs to associate with F-actin, all these variants should scaffold cellular components to the actin cytoskeleton (Ref. 66). The tissue specific expression of the CHD variants suggests that they are likely to play fundamental roles in tissue-specific actin-mediated signalling/ scaffolding events. This may contribute to determining the morphological structure of different cell lines and potentially regulate how different cells adapt the actin cytoskeleton for migration and adherence in vivo.

The involvement of $\mathrm{CHD}$ variants in actin mechanics has been identified in $\mathrm{p} 56 \mathrm{CH}^{\mathrm{Nesp} 1}$ and $\mathrm{p} 32 \mathrm{CH}^{\mathrm{Nesp} 2}$, the only nesprin $\mathrm{CHD}$ variants to be fully cloned and expressed into cells to date (Ref. 18). p32 $\mathrm{CH}^{\text {Nesp2 }}$ expression was limited to leukocytes and U2OS cells, localising to focal adhesions in the latter and implicating it in cell adhesion and migration. p56 $\mathrm{CH}^{\mathrm{Nesp} 1}$, composed of both nesprin-1 CHDs and a single $\mathrm{SR}$, appears to be ubiquitously expressed in all tissues and cells examined, however displays differential localisations in primary and transformed cell lines. In primary human dermal fibroblasts (HDFs), p56 $\mathrm{CH}^{\mathrm{Nesp} 1}$ localised to actin stress fibres, suggesting that it could scaffold cellular components to actin via its single SR. However, in U2OS cells, the protein appears to have nuclear functions, based on its nucleolar localisation. The presence of actin polymers within the nucleus and the structural motifs present in $\mathrm{p} 56 \mathrm{CH}^{\text {Nesp1 }}$ suggest it may function as a nucleolar scaffold in U2OS cells (Refs 70, 71). Nuclear actin appears to play fundamental roles in transcription mediated by all three RNA polymerases, chromatin

Accession information: doi:10.1017/erm.2013.6; Vol. 15; e5; July 2013 (C) Cambridge University Press 2013. Re-use permitted under a Creative Commons Licence - by-nc-sa. 
Figure 4. Potential nesprin-1 and nesprin-2 calponin homology domain (CHD) variants. By combining all the identified $3^{\prime}$ UTRs with the nesprin-1 giant and nesprin-2 giant 5'UTRs, up to 14 different nesprin-1 and 5 different nesprin-2 CHD containing variants can be translated. As with the Klarsicht, ANC-1 and Syne Homology (KASH) variants in Fig. 3, many of the large variants are hypothetical and yet to be validated at the mRNA and protein level. However, the tissue specific expression of the $3^{\prime}$ UTRs suggests that these variants are likely to be highly tissue specific. Therefore, the cells CHD variant expression profile may play a role in determining cell shape, rate of cell migration and contribute to its actin-mediated signalling/ scaffolding events.

remodelling, pre-mRNA processing and gene movement, implicating $\mathrm{p} 56 \mathrm{CH}^{\mathrm{Nesp} 1}$ in any number of nuclear actin mediated events (Ref. 72). The reasons behind the differential sub-cellular localisations currently remains unclear, but could partially be regulated by heterogeneous availability of the actin cytoskeleton in different cell types. Embedded within the nesprin-1 CHDs are two nuclear localisation signals which may be utilised in cells with low F-actin levels, such as U2OS cells, but not in structural cells such as HDFs where there is abundant cytoplasmic actin available for binding. Alternatively, p56 $\mathrm{CH}^{\mathrm{Nesp} 1}$ could display actin localisation in primary cells and relocalise to the nucleolus when cells become transformed during cancer development. Whether this change in localisation results in 
remodelling of nuclear actin polymers, resulting in misregulated nuclear-actin processes such as transcription, would make an interesting hypothesis.

Previously, Drop-1, a CHD nesprin-1 isoform composed of multiple SRs, has been identified as an isoform to be significantly down regulated in multiple cancers (Ref. 73). This study, along with the localisation changes observed with

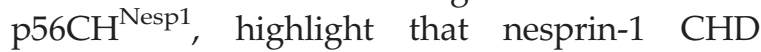
isoforms may be disrupted in cancers and could be classified as potential hallmarks of cellular transformation.

\section{SR isoforms}

To date, a greater number of alternate $5^{\prime}$ UTRs and 3'UTRs have been identified for nesprin-1 relative to nesprin-2, implying nesprin- 1 has the potential to generate a greater number of diverse isoforms. The positioning of the $5^{\prime}$ UTRs and $3^{\prime}$ UTRs across their genes suggests that nesprin-2 does not have the capacity to generate SR-only variants to the same extent as nesprin-1. With the 5'UTRs towards the end of the nesprin-2 gene and the 3'UTRs towards the beginning, nesprin-2 is more likely to generate KASH domain and CHD variants rather than those composed solely of SRs (Ref. 18). This suggests that nesprin-2 is prone to exert scaffolding functions at the NE and function in scaffolding protein complexes to F-actin rather than play roles in other subcellular compartments. However, by utilising the N2-3'E115 3'UTR or removing exons 111 and 112 by alternative splicing, two novel mechanisms exist which allow KASH-less and CHD-less nesprin-2 variants to be created. Indeed, a KASH-less nesprin-2 $\beta$ variant in vascular smooth muscle cells (VSMCs), likely to be generated through removal of exons 111 and 112 as detected previously, acts as a nuclear scaffold for ERK1/2 signalling pathways at PML nuclear bodies (Refs 18,69). When this complex was disrupted using siRNA-mediated nesprin-2 knockdown, ERK 1/2 was displaced from PML bodies and an enhanced ERK1/2 activity was observed, resulting in increased proliferation of VSMCs.

With nesprin-1 UTRs more randomly scattered along its gene, the combinations of nesprin-1 $5^{\prime}$ UTRs and 3'UTRs are potentially unlimited and would allow generation of a diverse spectrum of SR variants with specialised tissue specific scaffolding functions. A $56 \mathrm{kDa}$ Golgi-localised spectrin-repeat containing protein (GSRP-56) and candidate plasticity gene 2 (CPG2) were the first tissue-specific SR only nesprin-1 variants identified. GSRP-56 was identified in a yeast-2hybrid screen as a novel binding partner for transient receptor potential cation channel, vanilloid family type 2 (TRPV2) (Ref. 68). Endogenous GSRP-56 was detected and labelled the Golgi apparatus in myoblasts, myotubes and primary cardiomyocytes. When ectopically expressed in HEK293 cells, GSRP-56 formed large aggregates that surrounded the Golgi apparatus and induced Golgi area expansion, suggesting GSRP-56 may be a scaffold for the Golgi that regulates its structure (Ref. 68). CPG2 was first identified as a brain and neuron specific isoform which contributed to the long-term plasticity; the ability of neurons to stably alter their phenotype in response to various stimuli such as glutamate receptor activation and exposure to light (Refs 74, 75). Endogenous CPG2 localised to the cytosol, ER and postsynaptic endocytic zone surrounding dendritic spines in vitro, suggesting that CPG2 could participate in clathrin-mediated uptake and recycling of chemokine receptors (Ref. 76). In agreement with this function, knockdown of CPG2 led to an increase in the number of post-synaptic glutamate receptors with a concomitant increase of clathrin coated vesicles within dendritic arms, indicating that CPG2 is necessary for receptor internalisation and vesicle clearance (Ref. 76). Interestingly, knockdown of CPG2 decreased spine size, implying it may also regulate dendritic spine structure and morphology.

More recently, we identified multiple 5'UTRs and $3^{\prime}$ UTRs spread between exons 83 and 90 of the nesprin-1 gene, highlighting it as a hotspot region for the generation of multiple small nesprin SR isoforms. Indeed, five alternative transcripts could be detected in a tissue specific manner by alternatively combining the multiple UTRs (Fig. 5) (Ref. 18). p31 $1^{\text {Nesp1 }}, \mathrm{p} 23^{\text {Nesp1 }}$ and p12 ${ }^{\text {Nesp1 }}$ terminated with the N1-3'E87 3'UTR and all localised to the nucleolus when transfected into HDFs. Interestingly, the largest variant, $\mathrm{p} 31^{\text {Nesp1, }}$ localised to the nucleolus without disrupting nucleolar morphology when transfected into HDFs, whereas the N-terminally truncated $\mathrm{p} 23^{\mathrm{Nesp} 1}$ and $\mathrm{p} 12^{\mathrm{Nesp} 1}$ caused fibrillarin to re-distribute into nucleolar cap structures. This data implies $\mathrm{p} 31^{\mathrm{Nesp} 1}$ is a

Accession information: doi:10.1017/erm.2013.6; Vol. 15; e5; July 2013 (c) Cambridge University Press 2013. Re-use permitted under a Creative Commons Licence - by-nc-sa. 


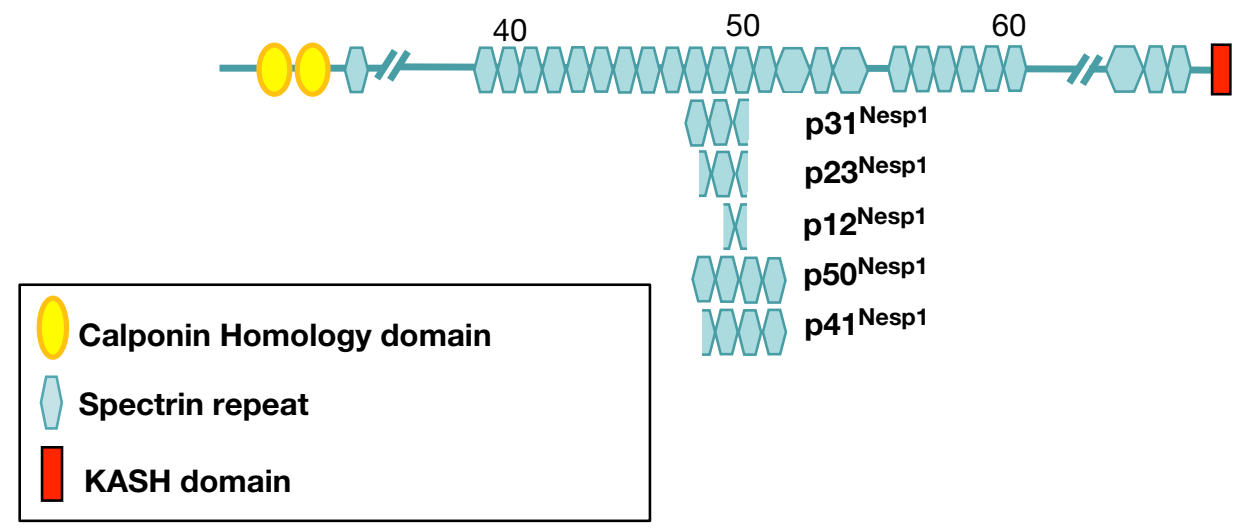

Figure 5. Spectrin repeat (SR)-only nesprin-1 isoforms generated from UTRs located between exons 83 and 90 of the nesprin-1 gene. Nesprin-1 isoforms $p 31^{\text {Nesp } 1}, p 23^{\text {Nesp } 1}, p 12^{\text {Nesp } 1}, p 50^{\text {Nesp } 1}$ and $p 41^{\text {Nesp } 1}$ are isoforms generated through alternative transcription using the three $5^{\prime}$ UTRs and two $3^{\prime}$ UTRs located between exons 83 and 90 .

potential nucleolar scaffold where the N-terminal SRs of the protein are critical for maintaining nucleolar structure in HDFs, which can be disrupted upon the overexpression of the Nterminally truncated $\mathrm{p} 23^{\text {Nesp1 }}$ or $\mathrm{p} 12^{\text {Nesp1 }}$ dominant negative isoforms. Interestingly, these variants were expressed in a tissue-specific manner, with only one of the three isoforms detected in each of the multiple tissues examined. Although the current function of these nucleolar isoforms are unknown, their tissue specificity highlights further the possibility that nesprin isoforms may act to fine tune tissue-specific cellular functions, including nucleolar functions. In contrast, when transfected into U2OS cells, all three variants localised diffusively within the cytosol, implying they have no nuclear functions in certain cell types. The ubiquitously expressed $\mathrm{p} 50^{\mathrm{Nesp} 1}$ localised to and bundled MTs when transfected into U2OS cells, suggesting it may be a Microtubule Associated Protein (MAP) capable of linking protein complexes to the MT network and acting as a MT scaffold.

\section{Nesprins and disease}

Multiple nesprin mutations have been identified that are likely to be included in a multitude of nesprin isoforms. For example, ARCA1 mutations in the nesprin-1 gene that produce premature termination codons, are likely to result in truncations of multiple nesprin isoforms and affect a range of nesprin-associated tissue specific scaffolds (Ref. 20). Similarly, a nesprin-4 mutation resulting in pre-mature termination has recently been reported to be causal for hearing loss. Disruption of nesprin-4 LINCs and nuclear migration in hair cells are thought to be the underlying cause, as shown convincingly with nesprin-4 $\mathrm{KO}$ mice (Ref. 77).

Mutations in lamin A/C and nesprin-1 have been renowned for causing dominant cardiomyopathies. A patient with a missense mutation near the KASH domain of nesprin-1 developed severe DCM requiring cardiac transplantation. Fibroblasts from this individual had increased expression of nesprin- $1 \alpha$ and lamins $A$ and $C$, indicating changes in nuclear lamina complexes (Ref. 22). These findings mirror what has been described from lamin A/C mutations, suggesting the importance of an intact nuclear lamina and LINC complex for a normal functioning heart (Ref. 78).

Heterozygous missense mutations have been identified in nesprin-1 and nesprin-2 when performing DNA screens on patients with EDMD or EDMD-like phenotypes. Fibroblasts from these patients exhibited nuclear morphology defects, mislocalised emerin and SUN-2 and impaired nesprin/emerin/lamin interactions, suggesting defective LINC complexes in these patients are the underlying cause of EDMD. siRNA-mediated nesprin-1 or nesprin-2 knockdown in normal fibroblasts reproduced the nuclear morphological changes and mislocalisation of emerin and Sun-2 observed in patient fibroblasts (Ref. 19). Later studies demonstrated that one of the nesprin-1

Accession information: doi:10.1017/erm.2013.6; Vol. 15; e5; July 2013

(C) Cambridge University Press 2013. Re-use permitted under a Creative Commons Licence - by-nc-sa. 
mutant-derived EDMD primary fibroblast cells were less adhesive, migrated slower in a wound healing assay and were more susceptible to senescence (Ref. 79). Similar localisation of emerin and SUN-2 are seen in EDMD patients carrying mutations in the INM transmembrane LUMA protein (Refs 80, 81). Although no interaction between LUMA and nesprins have been observed, the possibility of LUMA interacting with smaller KASH isoforms located at the INM is a strong possibility; especially considering mutations in both proteins cause EDMD and cardiomyopathies. Point mutations in nesprins are also casual for other diseases including $\mathrm{AMC}$, ARCA1 and bipolar disease. Therefore, it is not unreasonable to assume that these patients may have disrupted tissue-specific nesprin scaffolds (Refs 19, 20, 21, 22, 23). Although clearly not understood, nesprins have misregulated isoform expression in certain cancers, which indicates two things (Refs 73, 82, 83, 84). Firstly, nesprin associated complexes may be disrupted when other cellular signalling pathways are hampered, particularly those which control nesprin gene expression. Therefore, emphasis on identifying signalling pathways which govern gene expression and splicing of different nesprin isoforms is of utmost importance. Clearly, this is a significant factor, not only for aiding cancer therapy, but also for cellular differentiation and development where nesprins change isoform expression profiles (Refs 18, 48, 85). Secondly, nesprin isoform expression could be misregulated in a host of other diseases of the cardiovascular, muscular and neural systems. Indeed, nesprin-1 and nesprin-2 were originally discovered as two genes to be significantly down regulated in a cDNA screen when looking for VSMC differentiation markers, indicating a reduction of nesprin expression in the vasculature may promote atherosclerosis (Refs 14, 86). Therefore, the identification of isoform specific binding partners are fundamental in providing clues to the potential nesprin associated complexes and signalling pathways which are disrupted in nesprin associated diseases.

\section{Concluding remarks and future directions surrounding nesprin biology}

Over the last decade, our understanding of nesprins as NE-cytoskeletal couplers has increased considerably. However, there still remains a large uncertainty about the plethora of functions for nesprins, particularly now that it is clear they produce a diverse spectrum of tissue-specific isoforms with scaffolding roles throughout the cell (Fig. 6). To date, only a small number of nesprin isoforms have been cloned or described. Future work should involve a more in-depth study characterising each of the individual isoforms by generating appropriate tools such as isoform-specific antibodies and siRNAs, and suitable $\mathrm{KO}$ and transgenic animal models to elucidate the function of the individual endogenous isoforms in vivo. The lack of isoform specific sequences in many of the nesprin variants makes it difficult to design antibodies targeting a single isoform. However, the sequences created through intron run in, to initiate and terminate groups of nesprin variants, can generate unique N-terminal and C-terminal peptide sequences absent in the giant full-length variants. These may provide suitable sequences for generating antibodies for groups of isoforms initiating and terminating with specific sites. For example, N1-3'E87 and N1$3^{\prime} \mathrm{E} 90$ terminate with 10 and 8 unique amino acids, respectively, that are absent in variants terminating with other $3^{\prime}$ UTRs. Although this cannot be used to target any specific isoform, it will drastically reduce the number of isoforms detected when compared with the current crop of available antibodies. A similar approach will also be needed for designing siRNAs, where sequences within individual UTRs may need to be targeted. To date, only a limited number of nesprin animal models have been created, primarily examining the function of the LINC complex through disruption of KASH variants. Future work should focus on designing animal models that disrupt KASH-less variants, possibly by removing exon sequences, which encode for SRs present within the central rod region of nesprins.

The primary function of nesprins as cellular scaffolds and linkers suggests more needs to be done in identifying nesprin interacting proteins. To date, many nesprin binding partners have been identified; however, these are likely to represent only a small proportion of total interactors. More importantly, most binding partners are yet to be mapped to specific isoforms. Whether a protein which interacts with a specific SR in one variant is capable of interacting with the same SR in additional 


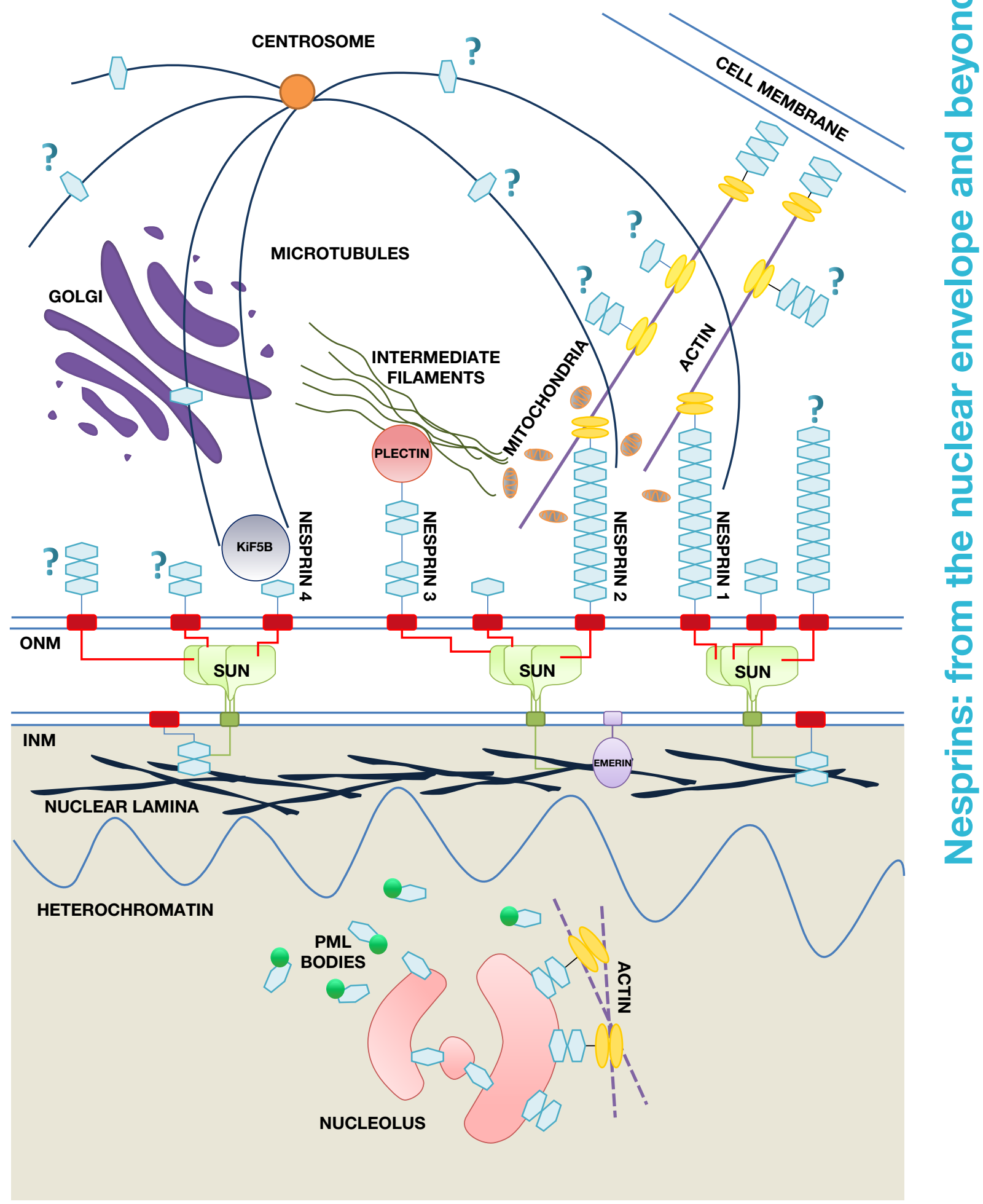

Figure 6. Nesprins act as linkers and provide scaffolds for multiple sub-cellular compartments. (See next page for legend.) 
variants is yet to be examined. There is the possibility that proteins may be unable to bind to a common SR in different variants through steric hindrance brought about by additional interacting proteins associating with nearby SRs in some variants. SRs are composed of three $\alpha$ helical bundles, where the first and last helices of an SR are also included in the final helix and the first helix of the two adjacent SRs, respectively. Therefore, depending on which start site is used to generate individual isoforms, or if any isoforms have altered alternative exon splicing, it is likely that the $\alpha$-helical structures utilised in a single SR could be altered in different variants, leading to a change in strength or abolishment, of protein binding to a common SR. Therefore, the identification of isoform specific binding partners, either from yeast-2-hybrid screens or pull-down experiments using exogenous tagged isoforms, is a necessity if the true extent of nesprin scaffolding is to be elucidated. Furthermore, binding partners from multiple tissues and cell lines may need to be obtained, given certain nesprin variants have distinctive localisations and therefore probable altered scaffolding functions in different cell lines.

\section{Acknowledgements and funding}

The authors would like to acknowledge Miss Aisling Williams for her excellent construction of Figures 2 and 6. This work was supported by the British Heart Foundation (BHF) and Medical Research Council (MRC).

\section{References}

1 Coulombe, P.A. and Wong, P. (2004) Cytoplasmic intermediate filaments revealed as dynamic and multipurpose scaffolds. Nature Cell Biology 6, 699-706

2 Rodriguez, O.C. et al. (2003) Conserved microtubule-actin interactions in cell movement and morphogenesis. Nature Cell Biology 5, 599-609

3 Yan, Y. et al. (1993) Crystal structure of the repetitive segments of spectrin. Science 262, 2027-2030

4 Parry, D.A., Dixon, T.W. and Cohen, C. (1992) Analysis of the three-alpha-helix motif in the spectrin superfamily of proteins. Biophysical Journal 61, 858-867

5 Djinovic-Carugo, K. et al. (1999) Structure of the alpha-actinin rod: molecular basis for cross-linking of actin filaments. Cell 98, 537-546

6 Djinovic-Carugo, K. et al. (2002) The spectrin repeat: a structural platform for cytoskeletal protein assemblies. FEBS Letters 513, 119-123

7 Leung, C.L., Green, K.J. and Liem, R.K. (2002) Plakins: a family of versatile cytolinker proteins. Trends in Cell Biology 12, 37-45

8 Stankewich, M.C. et al. (1998) A widely expressed betaIII spectrin associated with Golgi and cytoplasmic vesicles. Proceedings of the National Academy of Sciences of the United States of America 95, 14158-14163

9 Wang, D.S. and Shaw, G. (1995) The association of the C-terminal region of beta I sigma II spectrin to brain membranes is mediated by a PH domain, does not require membrane proteins, and coincides with a inositol-1,4,5 triphosphate binding site. Biochemical and Biophysical Research Communications 217, 608-615

10 Das, A. et al. (2008) Unexpected complexity in the mechanisms that target assembly of the spectrin cytoskeleton. Journal of Biological Chemistry 283, 12643-12653

Figure 6. Nesprins act as linkers and provide scaffolds for multiple sub-cellular compartments. (Legend; see previous page for figure.) Traditionally, the nesprins are known for their linker of nucleoskeleton to cytoskeleton (LINC) complex functions in linking the nuclear envelope (NE) to components of the cytoskeleton. However, through alternative transcription the nesprins are capable of generating multiple tissue specific isoforms that localise to multiple sub-cellular compartments. Klarsicht, ANC-1 and Syne Homology (KASH) domain isoforms can localise to the inner nuclear membrane (INM) and/or the outer nuclear membrane (ONM). At the INM, they interact with components of the nuclear lamina, including lamin $\mathrm{A} / \mathrm{C}$ and emerin. On the ONM, they interact directly or indirectly with all 3 major cytoskeletal filaments; Factin (Nesprin-1 and -2), intermediate filaments (IFs) (Nesprin-3 via plectin) and microtubules (MTs) (Nesprin-4 via kif5b and nesprin-1/-2 via dynein and kinesin-1). Additional nesprin-1 and nesprin-2 KASH variants on the ONM may link other cytosolic protein complexes or organelles, such as the Golgi or mitochondria, to the NE. KASH-less nesprin variants have been identified in multiple cytoplasmic and nuclear compartments including focal adhesions, F-actin, the Golgi, microtubules, promyelocytic (PML) bodies and the nucleolus. Question marks represent unidentified protein complexes, organelles or functions associated with various isoforms.

Accession information: doi:10.1017/erm.2013.6; Vol. 15; e5; July 2013

(c) Cambridge University Press 2013. Re-use permitted under a Creative Commons Licence - by-nc-sa. 
11 Ibraghimov-Beskrovnaya, O. et al. (1992) Primary structure of dystrophin-associated glycoproteins linking dystrophin to the extracellular matrix. Nature 355, 696-702

12 Sun, D., Leung, C.L. and Liem, R.K. (2001) Characterization of the microtubule binding domain of microtubule actin crosslinking factor (MACF): identification of a novel group of microtubule associated proteins. Journal of Cell Science 114(Pt 1), 161-172

13 Leung, C.L. et al. (1999) Microtubule actin crosslinking factor (MACF): a hybrid of dystonin and dystrophin that can interact with the actin and microtubule cytoskeletons. Journal of Cell Biology 147, 1275-1286

14 Zhang, Q. et al. (2001) Nesprins: a novel family of spectrin-repeat-containing proteins that localize to the nuclear membrane in multiple tissues. Journal of Cell Science 114(Pt 24), 4485-4498

15 Zhang, Q. et al. (2002) The nesprins are giant actinbinding proteins, orthologous to Drosophilamelanogaster muscle protein MSP-300. Genomics 80, 473-481

16 Wilhelmsen, K. et al. (2005) Nesprin-3, a novel outer nuclear membrane protein, associates with the cytoskeletal linker protein plectin. Journal of Cell Biology 171, 799-810

17 Roux, K.J. et al. (2009) Nesprin 4 is an outer nuclear membrane protein that can induce kinesin-mediated cell polarization. Proceedings of the National Academy of Sciences of the United States of America 106, 2194-2199

18 Rajgor, D. et al. (2012) Multiple novel nesprin-1 and nesprin-2 variants act as versatile tissue-specific intracellular scaffolds. PLoS ONE 7, e40098

19 Zhang, Q. et al. (2007) Nesprin-1 and -2 are involved in the pathogenesis of Emery Dreifuss muscular dystrophy and are critical for nuclear envelope integrity. Human Molecular Genetics 16, 2816-2833

20 Gros-Louis, F. et al. (2007) Mutations in SYNE1 lead to a newly discovered form of autosomal recessive cerebellar ataxia. Nature Genetics 39, 80-85

21 Attali, R. et al. (2009) Mutation of SYNE-1, encoding an essential component of the nuclear lamina, is responsible for autosomal recessive arthrogryposis. Human Molecular Genetics 18, 3462-3469

22 Puckelwartz, M.J. et al. (2010) Nesprin-1 mutations in human and murine cardiomyopathy. Journal of Molecular and Cellular Cardiology 48, 600-608

23 Green, E.K. et al. (2012) Association at SYNE1 in both bipolar disorder and recurrent major depression. Molecular Psychiatry 18, 614-7
24 Gerace, L. and Huber, M.D. (2012) Nuclear lamina at the crossroads of the cytoplasm and nucleus. Journal of Structural Biology 177, 24-31

25 Schirmer, E.C., Florens, L., Guan, T., Yates, J.R. 3rdGerace, L. (2003) Nuclear membrane proteins with potential disease links found by subtractive proteomics. Science 301, 1380-1382

26 Korfali, N. et al. (2012) The nuclear envelope proteome differs notably between tissues. Nucleus 3 , 552-564

27 Gruenbaum, Y. et al. (2005) The nuclear lamina comes of age. Nature Reviews. Molecular Cell Biology 6, 21-31

28 Burke, B. and Stewart, C.L. (2013) The nuclear lamins: flexibility in function. Nature Reviews. Molecular Cell Biology 14, 13-24

29 Sosa, B.A. et al. (2012) LINC complexes form by binding of three KASH peptides to domain interfaces of trimeric SUN proteins. Cell 149, 1035-1047

30 Zhou, Z. et al. (2012) Structure of Sad1-UNC84 homology (SUN) domain defines features of molecular bridge in nuclear envelope. Journal of Biological Chemistry 287, 5317-5326

31 Haque, F. et al. (2006) SUN1 interacts with nuclear lamin A and cytoplasmic nesprins to provide a physical connection between the nuclear lamina and the cytoskeleton. Molecular and Cellular Biology 26, 3738-3751

32 Crisp, M. et al. (2006) Coupling of the nucleus and cytoplasm: role of the LINC complex. Journal of Cell Biology 172, 41-53

33 McGee, M.D. et al. (2006) UNC-83 IS a KASH protein required for nuclear migration and is recruited to the outer nuclear membrane by a physical interaction with the SUN protein UNC-84. Molecular Biology of the Cell 17, 1790-1801

34 Starr, D.A. and Fischer, J.A. (2005) KASH 'n Karry: the KASH domain family of cargo-specific cytoskeletal adaptor proteins. Bioessays 27, 1136-1146

35 Stewart-Hutchinson, P.J. et al. (2008) Structural requirements for the assembly of LINC complexes and their function in cellular mechanical stiffness. Experimental Cell Research 314, 1892-1905

36 Mislow, J.M. et al. (2002) Nesprin-1alpha selfassociates and binds directly to emerin and lamin A in vitro. FEBS Letters 525, 135-140

37 Pare, G.C. et al. (2005) Nesprin-1alpha contributes to the targeting of mAKAP to the cardiac myocyte nuclear envelope. Experimental Cell Research 303, 388-399

38 Wheeler, M.A. et al. (2007) Distinct functional domains in nesprin-1alpha and nesprin-2beta bind 
directly to emerin and both interactions are disrupted in X-linked Emery-Dreifuss muscular dystrophy. Experimental Cell Research 313, 2845-2857

39 Haque, F. et al. (2010) Mammalian SUN protein interaction networks at the inner nuclear membrane and their role in laminopathy disease processes. Journal of Biological Chemistry 285, 3487-3498

$40 \mathrm{Lu}, \mathrm{W}$. et al. (2012) Nesprin interchain associations control nuclear size. Cellular and Molecular Life Sciences 69, 3493-509

41 Taranum, S. et al. (2012) Cytoskeletal interactions at the nuclear envelope mediated by nesprins. International Journal of Cell Biology 2012, 736524

42 Lombardi, M.L. et al. (2011) The interaction between nesprins and sun proteins at the nuclear envelope is critical for force transmission between the nucleus and cytoskeleton. Journal of Biological Chemistry 286, 26743-26753

43 Grady, R.M. et al. (2005) Syne proteins anchor muscle nuclei at the neuromuscular junction. Proceedings of the National Academy of Sciences of the United States of America 102, 4359-4364

44 Zhang, X. et al. (2007) Syne-1 and Syne-2 play crucial roles in myonuclear anchorage and motor neuron innervation. Development 134, 901-908

45 Anno, T., Sakamoto, N. and Sato, M. (2012) Role of nesprin-1 in nuclear deformation in endothelial cells under static and uniaxial stretching conditions. Biochemical and Biophysical Research Communications 424, 94-99

46 Brosig, M. et al. (2010) Interfering with the connection between the nucleus and the cytoskeleton affects nuclear rotation, mechanotransduction and myogenesis. International Journal of Biochemistry and Cell Biology 42, 1717-1728

47 Chancellor, T.J. et al. (2010) Actomyosin tension exerted on the nucleus through nesprin-1 connections influences endothelial cell adhesion, migration, and cyclic strain-induced reorientation. Biophysical Journal 99, 115-123

48 Randles, K.N. et al. (2010) Nesprins, but not sun proteins, switch isoforms at the nuclear envelope during muscle development. Developmental Dynamics 239, 998-1009

49 Morgan, J.T. et al. (2011) Nesprin-3 regulates endothelial cell morphology, perinuclear cytoskeletal architecture, and flow-induced polarization. Molecular Biology of the Cell 22, 4324-4334
50 Zhang, J. et al. (2010) Nesprin 1 is critical for nuclear positioning and anchorage. Human Molecular Genetics 19, 329-341

51 Mellad, J.A., Warren, D.T. and Shanahan, C.M. (2011) Nesprins LINC the nucleus and cytoskeleton. Current Opinion in Cell Biology 23, 47-54

52 Warren, D.T. et al. (2005) Nesprins: intracellular scaffolds that maintain cell architecture and coordinate cell function? Expert Reviews in Molecular Medicine 7, 1-15

53 Starr, D.A. and Han, M. (2002) Role of ANC-1 in tethering nuclei to the actin cytoskeleton. Science, 298, 406-409

54 Rashmi, R.N. et al. (2012) The nuclear envelope protein Nesprin-2 has roles in cell proliferation and differentiation during wound healing. Nucleus 3, 172-186

55 Gonzalez, S. et al. (2011) Mechanotransduction and epigenetic control in autoimmune diseases. Autoimmunity Reviews 10, 175-179

56 Lelievre, S.A. (2009) Contributions of extracellular matrix signaling and tissue architecture to nuclear mechanisms and spatial organization of gene expression control. Biochimica et Biophysica Acta 1790, 925-935

57 Gieni, R.S. and Hendzel, M.J. (2008) Mechanotransduction from the ECM to the genome: are the pieces now in place? Journal of Cellular Biochemistry 104, 1964-1987

58 Zhang, Q. et al. (2005) Nesprin-2 is a multi-isomeric protein that binds lamin and emerin at the nuclear envelope and forms a subcellular network in skeletal muscle. Journal of Cell Science 118(Pt 4), 673-687

59 Smith, E.R. et al. (2011) Increased expression of Syne1/nesprin-1 facilitates nuclear envelope structure changes in embryonic stem cell differentiation. Developmental Dynamics 240, 2245-2255

60 Malone, C.J. et al. (1999) UNC-84 localizes to the nuclear envelope and is required for nuclear migration and anchoring during $C$. elegans development. Development 126, 3171-3181

$61 \mathrm{Yu}$, J. et al. (2006) The KASH domain protein MSP300 plays an essential role in nuclear anchoring during Drosophila oogenesis. Developmental Biology 289, 336-345

62 Zhang, X. et al. (2009) SUN1/2 and Syne/Nesprin-1/ 2 complexes connect centrosome to the nucleus during neurogenesis and neuronal migration in mice. Neuron 64, 173-187

63 Fan, J. and Beck, K.A. (2004) A role for the spectrin superfamily member Syne-1 and kinesin II in cytokinesis. Journal of Cell Science 117(Pt 4), 619-629 
64 Gough, L.L. et al. (2003) Golgi localization of Syne-1. Molecular Biology of the Cell 14, 2410-2424

65 Padmakumar, V.C. et al. (2004) Enaptin, a giant actinbinding protein, is an element of the nuclear membrane and the actin cytoskeleton. Experimental Cell Research 295, 330-339

66 Zhen, Y.Y. et al. (2002) NUANCE, a giant protein connecting the nucleus and actin cytoskeleton. Journal of Cell Science 115(Pt 15), 3207-3222

67 Apel, E.D. et al. (2000) Syne-1, a dystrophin- and Klarsicht-related protein associated with synaptic nuclei at the neuromuscular junction. Journal of Biological Chemistry 275, 31986-31995

68 Kobayashi, Y. et al. (2006) Identification and characterization of GSRP-56, a novel Golgi-localized spectrin repeat-containing protein. Experimental Cell Research 312, 3152-3164

69 Warren, D.T. et al. (2010) Novel nuclear nesprin-2 variants tether active extracellular signal-regulated MAPK1 and MAPK2 at promyelocytic leukemia protein nuclear bodies and act to regulate smooth muscle cell proliferation. Journal of Biological Chemistry 285, 1311-1320

70 de Lanerolle, P. and Serebryannyy, L. (2011) Nuclear actin and myosins: life without filaments. Nature Cell Biology 13, 1282-1288

71 de Lanerolle, P., Johnson, T. and Hofmann, W.A. (2005) Actin and myosin I in the nucleus: what next? Nature Structural and Molecular Biology 12, 742-746

72 Miyamoto, K. and Gurdon, J.B. (2012)

Transcriptional regulation and nuclear reprogramming: roles of nuclear actin and actinbinding proteins. Cellular and Molecular Life Sciences. 10.1007/s00018-012-1235-7

73 Marme, A. et al. (2008) Loss of Drop1 expression already at early tumor stages in a wide range of human carcinomas. International Journal of Cancer 123, 2048-2056

74 Nedivi, E. et al. (1993) Numerous candidate plasticity-related genes revealed by differential cDNA cloning. Nature 363, 718-722
75 Nedivi, E. et al. (1996) A set of genes expressed in response to light in the adult cerebral cortex and regulated during development. Proceedings of the National Academy of Sciences of the United States of America 93, 2048-2053

76 Cottrell, J.R. et al. (2004) CPG2: a brain- and synapsespecific protein that regulates the endocytosis of glutamate receptors. Neuron 44, 677-690

77 Horn, H.F. et al. (2013) The LINC complex is essential for hearing. Journal of Clinical Investigation 123, 740-50

78 Malhotra, R. and Mason, P.K. (2009) Lamin A/C deficiency as a cause of familial dilated cardiomyopathy. Current Opinion in Cardiology 24, 203-208

79 Taranum, S. et al. (2012) LINC complex alterations in DMD and EDMD/CMT fibroblasts. European Journal of Cell Biology 91, 614-628

80 Liang, W.C. et al. (2011) TMEM43 mutations in Emery-Dreifuss muscular dystrophy-related myopathy. Annals of Neurology 69, 1005-1013

81 Bengtsson, L. and Otto, H. (2008) LUMA interacts with emerin and influences its distribution at the inner nuclear membrane. Journal of Cell Science 121(Pt 4), 536-548

82 Schuebel, K.E. et al. (2007) Comparing the DNA hypermethylome with gene mutations in human colorectal cancer. PLoS Genet 3, 1709-1723

83 Tessema, M. and Belinsky, S.A. (2008) Mining the epigenome for methylated genes in lung cancer. Proceedings of the American Thoracic Society 5, 806-810

84 Tessema, M. et al. (2008) Promoter methylation of genes in and around the candidate lung cancer susceptibility locus 6q23-25. Cancer Research 68, $1707-1714$

85 Dawe, H.R. et al. (2009) Nesprin-2 interacts with meckelin and mediates ciliogenesis via remodelling of the actin cytoskeleton. Journal of Cell Science 122(Pt 15), 2716-2726

86 Shanahan, C.M., Weissberg, P.L. and Metcalfe, J.C. (1993) Isolation of gene markers of differentiated and proliferating vascular smooth muscle cells. Circulation Research 73, 193-204 


\section{Figures}

Figure 1. The nesprin family.

Figure 2. Nesprins link the nucleoskeleton to components of the cytoskeleton.

Figure 3. Potential nesprin-1 and nesprin-2 Klarsicht, ANC-1 and Syne Homology (KASH) variants.

Figure 4. Potential nesprin-1 and nesprin-2 calponin homology domain (CHD) variants.

Figure 5. Spectrin repeat (SR)-only nesprin-1 isoforms generated from UTRs located between exons 83 and 90 of the nesprin-1 gene.

Figure 6. Nesprins act as linkers and provide scaffolds for multiple sub-cellular compartments

\section{Citation details for this article}

Dipen Rajgor and Catherine M. Shanahan (2013) Nesprins: from the nuclear envelope and beyond. Expert Rev. Mol. Med. Vol. 15, e5, July 2013, doi:10.1017/erm.2013.6 